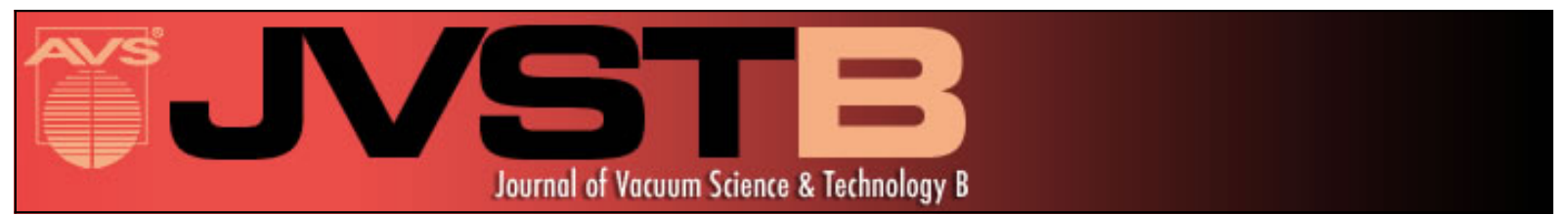

\title{
Numerical study of the laser-tip coupling in surface plasmon assisted stacked-double- gate field emitter arrays
}

Youngjin Oh, Anna Mustonen, Thomas Feurer, and Soichiro Tsujino

Citation: Journal of Vacuum Science \& Technology B 33, 03C112 (2015); doi: 10.1116/1.4915252

View online: http://dx.doi.org/10.1116/1.4915252

View Table of Contents: http://scitation.aip.org/content/avs/journal/jvstb/33/3?ver=pdfcov

Published by the AVS: Science \& Technology of Materials, Interfaces, and Processing

\section{Articles you may be interested in}

Highly collimated electron beams from double-gate field emitter arrays with large collimation gate apertures

Appl. Phys. Lett. 98, 061502 (2011); 10.1063/1.3551541

Transmission properties of surface plasmon polaritons and localized resonance in semiconductor hole arrays Appl. Phys. Lett. 97, 261111 (2010); 10.1063/1.3532111

Efficient high-current field emission from arrays of carbon nanotube columns

J. Vac. Sci. Technol. B 28, C2B14 (2010); 10.1116/1.3300062

Study of techniques for improving emission uniformity of gated $\mathrm{CuO}$ nanowire field emitter arrays

J. Vac. Sci. Technol. B 28, C2C45 (2010); 10.1116/1.3299065

Double-gate field emitters with planar carbon-nanoparticle cathodes: Simulation studies

J. Vac. Sci. Technol. B 22, 1303 (2004); 10.1116/1.1722066

\section{HDDEN}

\section{Instruments for Advanced Science}

Contact Hiden Analytical for further details: w www.HidenAnalytical.com E info@hiden.co.uk CLICK TO VIEW our product catalogue

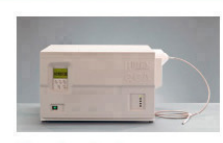

Gas Analysis

, dynamic measurement of reaction gas streams catalysis and thermal analysis molecular beam studies 1, fermentation environmental and ecological studies

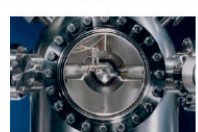

Surface Science

, UHVTPD

SIMS end point detection in ion beam etch

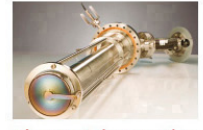

Plasma Diagnostics , plasma source characterization etch and deposition process reaction

analysis of neutral and radical species

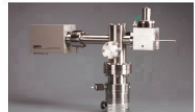

Vacuum Analysis , partial pressure measurement and control of process gases , eactive sputter process control vacuum coating process monitoring 


\title{
Numerical study of the laser-tip coupling in surface plasmon assisted stacked-double-gate field emitter arrays
}

\author{
Youngjin Oh and Anna Mustonen \\ Laboratory for Micro and Nanotechnology, Paul Scherrer Institute, Villigen-PSI 5232, Switzerland \\ Thomas Feurer \\ Institute of Applied Physics, University of Bern, Sidlerstrasse 5, Bern 3012, Switzerland \\ Soichiro Tsujino ${ }^{\text {a) }}$ \\ Laboratory for Micro and Nanotechnology, Paul Scherrer Institute, Villigen-PSI 5232, Switzerland
}

(Received 31 October 2014; accepted 3 March 2015; published 19 March 2015)

\begin{abstract}
Recently, sub-wavelength-pitch stacked double-gate metal nanotip arrays have been proposed to realize high current, high brightness electron bunches for ultrabright cathodes for x-ray free-electron laser applications. With the proposed device structure, ultrafast field emission of photoexcited electrons is efficiently driven by vertical incident near infrared laser pulses, via near field coupling of the surface plasmon polariton resonance of the gate electrodes with the nanotip apex. In this work, in order to gain insight in the underlying physical processes, the authors report detailed numerical studies of the proposed device. The results indicate the importance of the interaction of the doublelayer surface plasmon polariton, the position of the nanotip, as well as the incident angle of the near infrared laser pulses. (C) 2015 American Vacuum Society.
\end{abstract}

[http://dx.doi.org/10.1116/1.4915252]

\section{INTRODUCTION}

Considerable attention has been paid recently to metal nanotips excited by femtosecond near infrared laser pulses to generate high current, high brightness, and ultrafast electron pulses for advanced accelerator applications such as the compact $\mathrm{X}$-ray free electron lasers (X-FELs). ${ }^{1-8}$ With its low emittance and high electron charge, it may also be suited for $\mathrm{THz}$ vacuum electronic oscillator and amplifier cathodes 9,10 as well as for ultrafast electron microscopy. ${ }^{11-15}$ Recent experiments demonstrated generation of short electron bunches with up to $5 \mathrm{pC}$ bunch charge using an array of $1.2 \times 10^{5}$ metal nanotips with an array pitch of $5 \mu \mathrm{m} .{ }^{16,17}$ Further increase of the electron yield by a few orders of magnitude is still required to be compatible with $\mathrm{X}$-FEL requirements. Therefore, we proposed and theoretically analyzed the performance of sub-micron-pitch all metal stacked double-gate nanotip arrays, which promise an increased electron yield. The higher nanotip density can enhance the electron yield of the near infrared laser induced field emission if one can achieve the same degree of the nanotip apex excitation efficiency. We showed that this is indeed the case when including the surface-plasmon-polariton (SPP) resonance of the gate electrodes; from theoretical analysis we predicted that using such a double-gate field emitter array (FEA) with a $10^{6}$ nanotip array with $1 \mathrm{~mm}$ diameter, is sufficient to generate $200 \mathrm{pC}$ electron pulses with an intrinsic transverse beam emittance below $0.1 \mathrm{~mm}$ mrad when the FEA is excited by vertically incident near infrared laser pulses with the duration of $1-10 \mathrm{ps}$ and pulse energies of $\sim 0.1 \mathrm{~mJ}$.

However, the precise physical mechanisms and the quantitative relationships between the SPP resonance and the tiplight coupling are yet to be elucidated. Therefore, we report

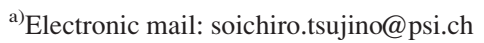

in this work the parametric study of the tip-light coupling in stacked-double-gate structures as well as the SPP resonance of the gate electrodes.

\section{MODELING}

The double-gate FEA structure we propose, Fig. 1, consists of sub-micron-pitch molybdenum nanotips with the copper double-gate electrodes. The double-gate electrode layers on top of the nanotips for the electron extraction and the beam collimation, ${ }^{18}$ act also to enhance the coupling of the laser pulses to the nanotip apexes ${ }^{3,4}$ at the same time. The optical field enhancement at the tip apexes is a result of the geometrical field enhancement ${ }^{17}$ and the near field coupling of the nanotip with the electric field of the resonantly excited SPP near the gate edge at the optical frequency determined by the dispersion of the surface-plasmon ${ }^{19,20}$ of the material and the period of the apertures. The enhanced optical transmission through subwavelength nanohole arrays via the SPP excitation, which was first reported by Ebbesen et al. as the extraordinary optical transmission (EOT), is commonly observed using $\mathrm{Au}$ or $\mathrm{Ag}$ since these have low optical attenuation in visible/near infrared range. ${ }^{21} \mathrm{We}$ propose to use copper since not only copper has the low optical attenuation but also it is a material commonly utilized for accelerator applications with the high acceleration electric field (up to $100 \mathrm{MV} / \mathrm{m}$ or higher) ${ }^{22}$ that is required for high brightness beam applications to prevent the space-charge degradation of the transverse beam emittance.

The stacked double-gate nanotip FEA used in the numerical modeling is depicted in Fig. 1. The molybdenum metal nanotips are modeled as $120 \mathrm{~nm}$ tall cone-shaped emitters with the tip apex radius of curvature of $5 \mathrm{~nm}$ and an array pitch of $750 \mathrm{~nm}$. The electron extraction gate electrode $G_{\text {ext }}$ and the beam collimation gate electrode $G_{\text {col }}$ are stacked on 


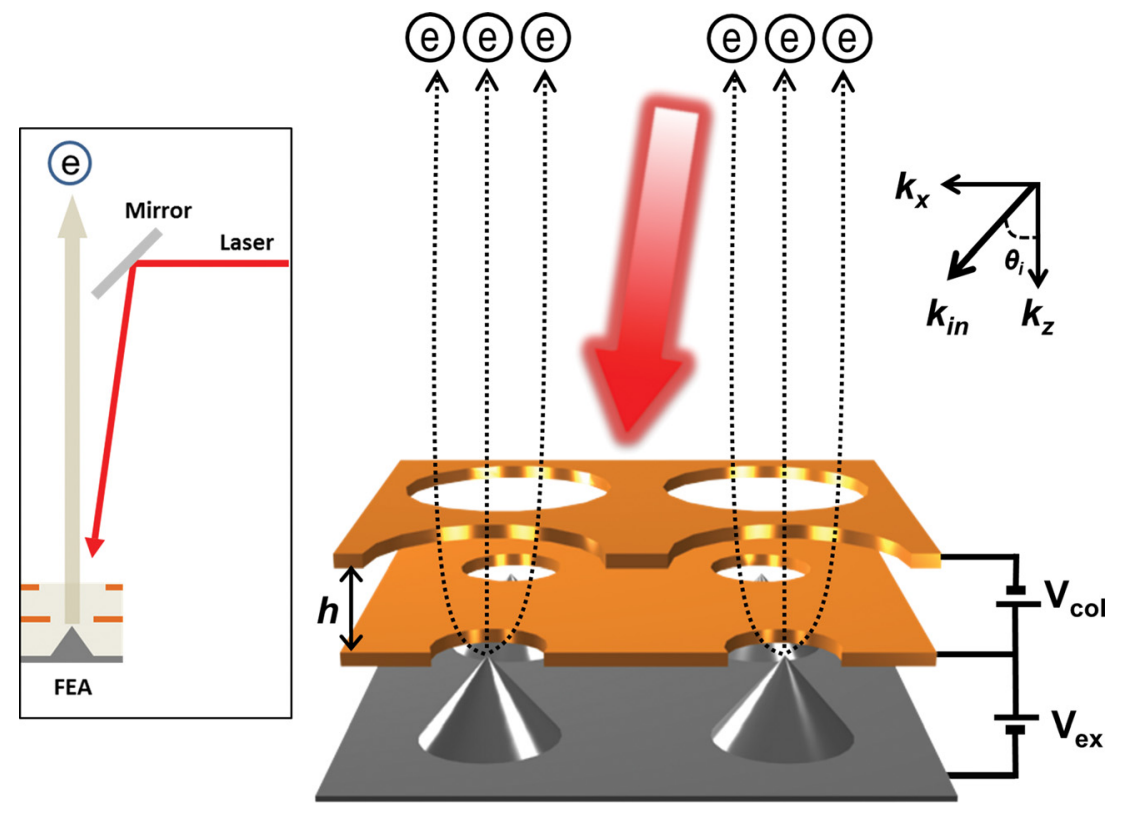

FIG. 1. (Color online) Schematic of the proposed stacked double-gate FEA excited by near infrared laser pulses.

top of the nanotip array. We assumed that the gates are $50 \mathrm{~nm}$-thick copper layers. We assumed a gate layer thickness equal to $50 \mathrm{~nm}$. The gate aperture diameter of $G_{\text {ext }}$ is equal to $200 \mathrm{~nm}$ and the gate aperture diameter of $G_{\mathrm{col}}$ is $600 \mathrm{~nm}$. The large $G_{\mathrm{col}}$ aperture structure is important to achieve maximum beam collimation with the minimal emission current reduction, see Ref. 18.

To simulate the electromagnetic field distribution of the device upon near infrared excitation, we used a threedimensional finite element electromagnetic solver (COMSOL Multiphysics). Optical constants of $\mathrm{Cu}$ and $\mathrm{Mo}$ were taken from Ref. 23. We assumed periodic (Floquet) boundary conditions in the transverse direction to simulate the array. The nanotip array was excited by a transverse magnetic plane wave with an incident angle of $\theta_{i}$. We additionally varied the separation $h$ between the $G_{\text {ex }}$ and $G_{\mathrm{col}}$ layers. The nanotip position is off-center to obtain a finite field enhancement at the emitter tip apex; $;^{3,6}$ e.g., a $30 \mathrm{~nm}$ shift resulted in a field enhancement of nearly a factor of 10 at the SPP resonance ${ }^{4}$ for vertical incident excitation. We also consider the zero offset case for different $\theta_{i}$.

In addition to the electromagnetic field distribution of the double-gate FEA structures, to study the quantitative relationship between the EOT and the enhancement of the optical electric field at the tip apexes, we also simulated optical transmission spectra of the single- and double-layer nanohole arrays without the nanotips, see Figs. 2(b) and 2(c), at vertical incident case. To simulate the far-field transmission of the structure, the out-going optical flux was integrated over a plane at $2 \mu \mathrm{m}$ below the hole layers.

\section{RESULTS AND DISCUSSION}

We first discuss the optical transmission spectrum through the nanohole arrays (without nanotips) for the single- and double-copper-layers with the same layer thicknesses and the hole period as the double-gate FEA in Fig. 1. Figure 2 shows the calculated results for the two single-layer nanohole array transmission spectra, one with an aperture diameter of $200 \mathrm{~nm}$ (same as $G_{\text {ext }}$ ) and another with an aperture diameter of $600 \mathrm{~nm}$ (same as $G_{\text {col }}$ ) exhibit an EOT resonance ${ }^{21}$ near $1.65 \mathrm{eV}$. Figures 2(b) and 2(c) show schematic of single- and doublelayer nanohole array, respectively. Even though the array pitch is identical, the transmission resonance peaks are observed at different photon energies tens of $\mathrm{meV}$ apart. This is ascribed to the fact that the EOT resonance is a combined effect of the SPP resonance and its transmission through the aperture that acts as a plasmonic waveguide. ${ }^{24,25}$ The order of magnitude higher resonant transmission for the $600 \mathrm{~nm}$ diameter hole array compared to the $200 \mathrm{~nm}$ diameter hole array is due to the larger area of the hole. ${ }^{26}$ Most interesting is the transmission spectrum of the double-layer nanohole array. The double-layer structure also shows the EOT-like transmission resonance. Its peak transmission is higher than the combined (multiplied) transmission of the two individual single-layer hole arrays. The resonance occurs again near $1.65 \mathrm{eV}$ but at a somewhat lower energy than the single-layer transmission peak position. The Fano dip at the higher photon energy side of the double-layer EOT resonance is also deeper than the single-layer spectra. These observations suggest that the SPP resonance and transmission of the doublelayer hole array is strongly affected by the near-field interaction of the SPP resonances of the two single-layer hole arrays. ${ }^{27}$ Figure 3(a) shows the variation of the double-layer EOT transmission spectrum when the separation $h$ between the layers is varied from 30 to $200 \mathrm{~nm}$. We found that the peak transmission and the width of the resonance monotonically increase with increasing $h$, while the resonant photon energy decreases at the same time. This seems to be related to the increasing mode strength of the internal SPP between the two metal layers with the increase of $h^{24}$ 

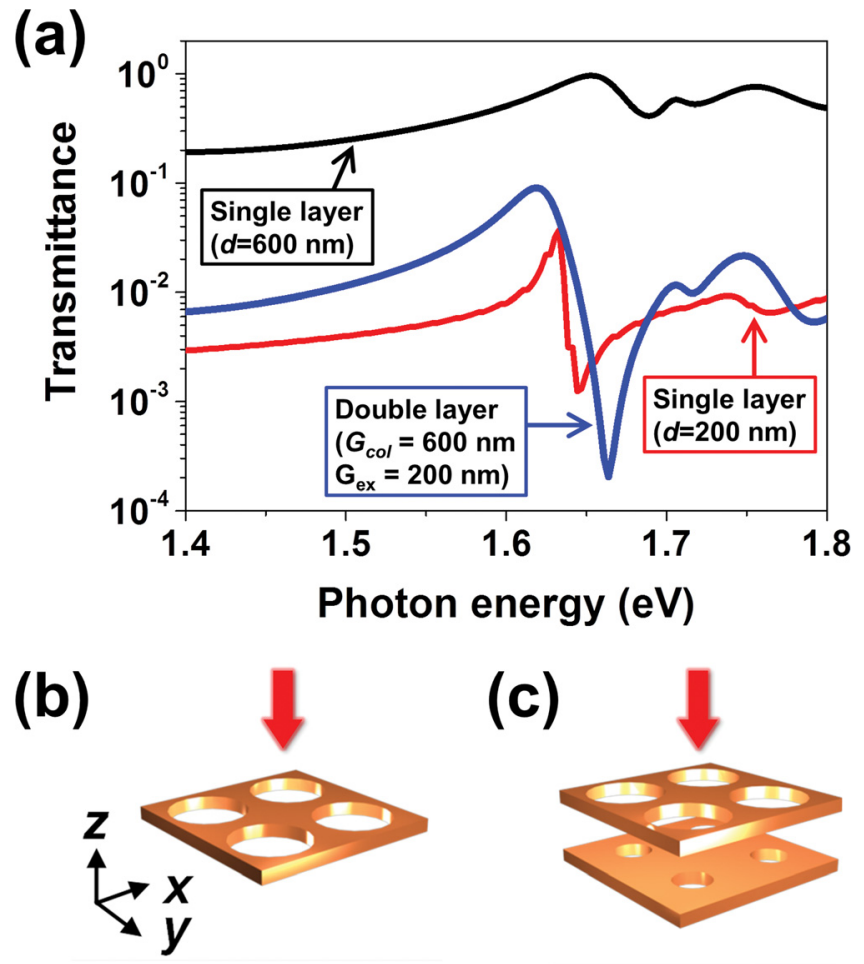

FIG. 2. (Color online) (a) Simulated optical transmission spectra of the copper single- and double-layer nanohole arrays with the pitch of the holes of $750 \mathrm{~nm}$. Two single-layer hole arrays have the diameter equal to 200 and $600 \mathrm{~nm}$, which are, respectively, same as the aperture diameter of $G_{\text {ext }}$ and $G_{\text {col }}$ of the proposed double-gate nanotip array (Fig. 1). The double-layer hole array is a stack of these two single-layer hole arrays separated by $120 \mathrm{~nm}$ (inset). The spectra exhibit the extraordinary optical transmission resonance via the excitation of the surface-plasmon-polariton of the singleand double-copper-layer, suggesting that, when used as the gate-layers for metal nanotip arrays as shown in Fig. 1, the near infrared optical excitation at the tip apex can be enhanced despite the subwavelength aperture diameters of the sub-micron-pitch nanotip arrays with a hole pitch of $750 \mathrm{~nm}$. The schematic drawings of the single- and double-layer hole arrays are depicted in (b) and (c), respectively. The transmission spectra were calculated in the vertical incidence case as indicated by the red arrows in (b) and (c).
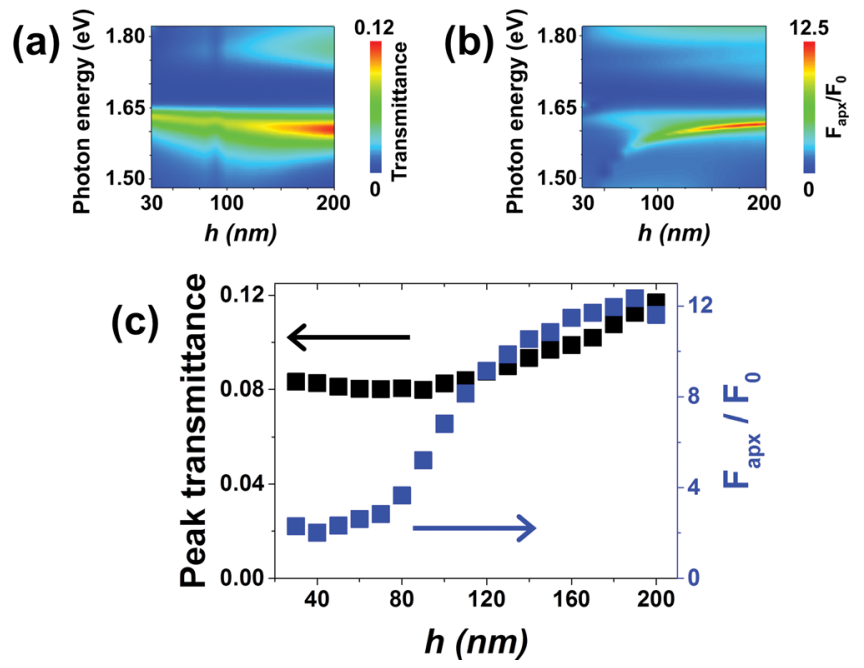

FIG. 3. (Color online) (a) Transmission spectra of the double-layer nanohole array at different layer separation $h$. (b) Spectra of the optical electric field at the apex $F_{\text {apx }}$ calculated at different $h$ for vertical incidence case. (c) The relation between $h$ and the peak transmission (left axis) and the peak $F_{\text {apx }}$ (right axis). (a)
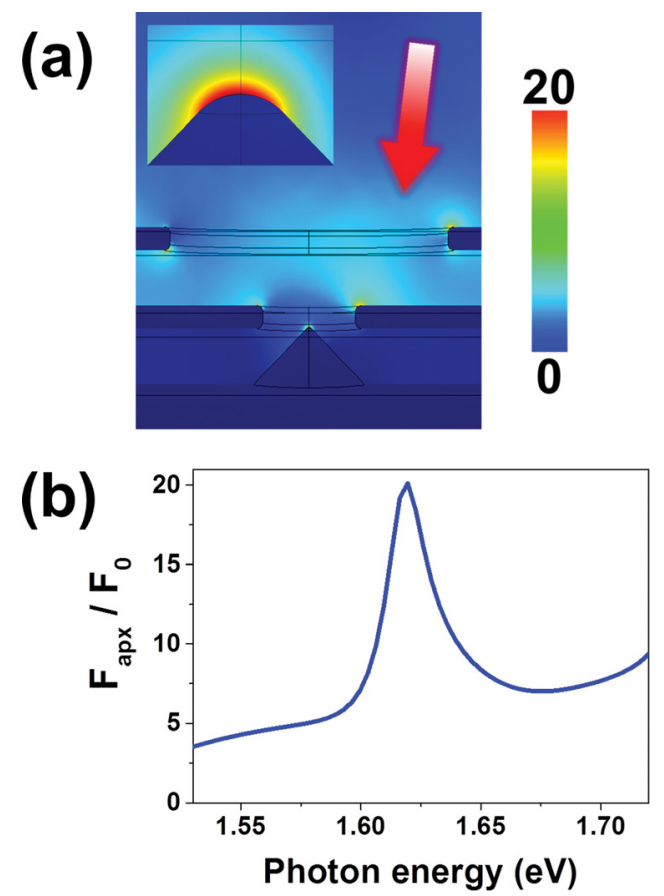

FIG. 4. (Color online) (a) Optical field distribution of the double $\mathrm{Cu}$ gate Mo nanotip structure for near infrared excitation at $1.60 \mathrm{eV}$ and $7^{\circ}$ incident angle (red arrow). Due to the finite incident angle, $F_{\text {apx }}$ is resonantly enhanced up to 20 (see inset) despite the fact that the nanotip is placed at the center of the gate apertures. (b) The optical frequency dependence of $F_{\text {apx }}$ with $7^{\circ}$ incident angle shows the resonance is at $1.60 \mathrm{eV}$ with the half width half maximum width of $\sim 19 \mathrm{meV}(\sim 28 \mathrm{THz})$.

Next, we studied the variation of the optical electric field $F_{\text {apx }}$ at the emitter tip apex in the double-gate FEA structure as $h$ is varied between 30 and $200 \mathrm{~nm}$. We show the result in Fig. 3(b) where we divided $F_{\text {apx }}$ by the optical electric field $F_{0}$ of the incident field. As summarized in Fig. 3(c), the enhancement given by $F_{\text {apx }} / F_{0}$ is substantial, close to $8-10$, only when $h$ is larger than $\sim 100 \mathrm{~nm}$. In the same $h$-range, the photon energy of the peak $F_{\text {apx }}$ is the same as that of the transmission resonance within $\sim 10 \mathrm{meV}$. However, different from the transmission peak, the peak photon energy of $F_{\text {apx }}$ monotonously decreases with the decrease of $h$. Note that the peak $F_{\text {apx }}$ increases more rapidly than the peak transmittance. This shows that to achieve a large peak $F_{\text {apx }}$ the details of the nearfield distribution around the $G_{\text {ex }}$ aperture have to be taken into account, which may sensitively depend on the detailed $G_{\text {ex }}$ edge shape as well as the subtle interaction and attenuation of the SPP field between the two layers. ${ }^{20}$ Further comprehensive study is required in the future to clarify this point.

Finally, we discuss the case when the excitation near infrared light is at oblique incidence. Our consideration so far was limited to the vertical incidence case, where the incident optical electric field has only a transverse component. We find a large $F_{\text {apx }}$ at the emitter tip apex in the vertical direction which is induced by the SPP resonance of the gate electrode when placing the nanotip at $\sim 30 \mathrm{~nm}$ off-center. In practical applications, however, it is desirable to have a small but finite incident angle as depicted in the left panel of Fig. 1. We therefore consider the case when the incident angle is $7^{\circ}$ as an example, which separates the electron beam and the laser coupling 
mirror by tens of millimeters when the mirror is inserted several hundred millimeter upstream from the cathode surface. As shown in Fig. 4, we found that a large $F_{\text {apx }}$ equal to $\sim 20$ can be achieved at the resonance condition even when the nanotip is placed at the center of the gate apertures. Apparently, the asymmetry of the SPP near-field at the $G_{\text {ex }}$ edge induced by the finite incident angle is sufficient to compensate for the symmetric position of the nanotip and to enhance $F_{\text {apx }}$ by a factor 2 larger than the vertical incident, shifted-tip case. By accounting for the factor $20 F_{\text {apx }}$ enhancement, the predicted electron yield of the device approaches $10^{-5}$, thus requiring near infrared pulse energies of $\sim 25 \mu \mathrm{J}$ to generate $\sim 200$ pC electron pulses from a $10^{6}$ nanotip array. The large resonance width of $18 \mathrm{meV}$ ( $\sim 28 \mathrm{THz}$ ) assures the assumed operation condition with the electron pulse duration of $1-10 \mathrm{ps}$.

\section{SUMMARY AND CONCLUSIONS}

We numerically studied the SPP resonance of the doublelayer nanohole array and its influence on the near infrared excitation efficiency of the nanotips in the double-gate structures. The comparison of the transmission spectra of the single- and double-layer nanohole arrays strongly suggests a major influence of the internal SPP field on the excitation of the nanotip apex that leads to a large $F_{\text {apx }}$ field. Moreover, by considering the more practically important case of a finite incident angle, we found that a larger $F_{\text {apx }}$ can be achieved by introducing $7^{\circ}$ incident angle for the double-gate structure without shifting the nanotip position from the gate aperture center. These results strongly suggests that to fully elucidate the physical mechanism of the enhanced tip-light interaction in the SPP-enhanced double-gate nanotip arrays, further comprehensive parametric studies investigating the SPP momentum dependence and the $G_{\text {ext }}$ edge shape dependence as well as experimental validation are required. These are the subjects of the on-going research.

\section{ACKNOWLEDGMENTS}

This work was partially supported by the Swiss National Science Foundation, Grant Nos. 200020_143428 and 2000021_147101.
${ }^{1}$ M. Dehler, A. Candel, and E. Gjonaj, J. Vac. Sci. Technol. B 24, 892 (2006).

${ }^{2}$ P. Helfenstein, E. Kirk, K. Jefimovs, T. Vogel, C. Escher, H.-W. Fink, and S. Tsujino, Appl. Phys. Lett. 98, 061502 (2011).

${ }^{3}$ A. Mustonen, P. Beaud, E. Kirk, T. Feurer, and S. Tsujino, Sci. Rep. 2, 915 (2012).

${ }^{4}$ P. Helfenstein, A. Mustonen, T. Feurer, and S. Tsujino, Appl. Phys. Express 6, 114301 (2013).

${ }^{5}$ B. D. Patterson et al., Chimia 68, 73 (2014).

${ }^{6}$ B. D. Patterson et al., New. J. Phys. 12, 035012 (2010).

${ }^{7}$ C. A. Brau, Nucl. Instrum. Methods Phys. Res. A 407, 1 (1998).

${ }^{8}$ W. S. Graves, F. X. Kärtner, D. E. Moncton, and P. Piot, Phys. Rev. Lett. 108, 263904 (2012).

${ }^{9}$ K. B. K. Teo et al., Nature 437, 968 (2005).

${ }^{10}$ H. Makishima, S. Miyano, H. Imura, J. Matsuoka, H. Takemura, and A. Okamoto, Appl. Surf. Sci. 146, 230 (1999).

${ }^{11}$ A. V. Crewe, J. Wall, and J. Langmore, Science 168, 1338 (1970).

${ }^{12}$ M. Kruger, M. Schenk, and P. Hommelhoff, Nature 475, 78 (2011).

${ }^{13}$ P. Hommelhoff, C. Kealhofer, and M. A. Kasevich, Phys. Rev. Lett. 97, 247402 (2006).

${ }^{14}$ C. C. Neacsu, S. Berweger, R. L. Olmon, L. V. Saraf, C. Ropers, and M. B. Raschke, Nano Lett. 10, 592 (2010).

${ }^{15} \mathrm{H}$. Yanagisawa, M. Hengsberger, D. Leuenberger, M. Klockner, C. Hafner, T. Greber, and J. Osterwalder, Phys. Rev. Lett. 107, 087601 (2011).

${ }^{16}$ S. Tsujino, P. Beaud, E. Kirk, T. Vogel, H. Sehr, J. Gobrecht, and A. Wrulich, Appl. Phys. Lett. 92, 193501 (2008).

${ }^{17}$ A. Mustonen, P. Beaud, E. Kirk, T. Feurer, and S. Tsujino, Appl. Phys. Lett. 99, 103504 (2011).

${ }^{18}$ P. Helfenstein, K. Jefimovs, E. Kirk, C. Escher, H.-W. Fink, and S. Tsujino, J. Appl. Phys. 113, 043306 (2013).

${ }^{19}$ C. Kittel and P. McEuen, Introduction to Solid State Physics, 8th ed. (Wiley, Hoboken, NJ, 2005).

${ }^{20}$ S. A. Maier, Plasmonics (Springer, New York, 2007).

${ }^{21}$ T. W. Ebbesen, H. J. Lezec, H. F. Ghaemi, T. Thio, and P. A. Wolff, Nature 391, 667 (1998).

${ }^{22}$ V. G. Tkachenko, A. I. Kondrashev, and I. N. Maksimchuk, Appl. Phys. B 98, 839 (2010).

${ }^{23}$ E. D. Palik, Handbook of Optical Constants of Solids (Academic, Orlando, 1985).

${ }^{24}$ R. Ortuño, C. García-Meca, F. J. Rodríguez-Fortuño, J. Martí, and A. Martínez, Phys. Rev. B 79, 075425 (2009).

${ }^{25}$ Z. H. Tang, R. W. Peng, Z. Wang, X. Wu, Y. J. Bao, Q. J. Wang, Z. J. Zhang, W. H. Sun, and M. Wang, Phys. Rev. B 76, 195405 (2007).

${ }^{26} \mathrm{H}$. A. Bethe, Phys. Rev. 66, 163 (1944).

${ }^{27}$ The resonance width of $\sim 0.1 \mathrm{eV}$ corresponding to $\sim 10 \mathrm{THz}$ is more than a factor of 10 larger than the required bandwidth of 1-10 ps laser pulses assumed for the operation of the proposed FEAs, see Ref. 4. 